\title{
EVALUACIÓN DE UNA INTERVENCIÓN COMUNITARIA POST DESASTRES EN LA COMUNIDAD SAN FRANCISCO DE SALES, GUATEMALA
}

ASSESSMENT OF A POST-DISASTER COMMUNITY INTERVENTION PLAN AT SAN FRANCISCO DE SALES POPULATION IN GUATEMALA

SANDRA E. LUNA 1 ; CARLOS TAGRE 1 ; ANDREA A B BOU I $_{1}$ ANNA SOPHÍA SALAZAR 1 ; DANIA HERRERA A $_{1}$ ADRIANA

AGUILAR ; MARILYN MARTÍNEZ

(UNIVERSIDAD RAFAEL LANDÍVAR, Ciudad de Guatemala, Guatemala)

\begin{abstract}
RESUMEN
Introducción: Durante los años 2012 y 2013 se realizaron intervenciones comunitarias en el municipio San Vicente Pacaya, departamento de Escuintla, Guatemala, destinadas a intervenir en las secuelas de la erupción del Volcán de Pacaya y de la entrada inmediata de la Tormenta Agatha el 2010. Objetivo: Evaluar el efecto individual y comunitario que tuvo el trabajo de intervención pos desastre realizado por estudiantes de Psicología en la comunidad San Francisco de Sales. Método: Se realizan evaluaciones individuales y grupos focales, utilizando como instrumentos de recolección de datos diarios de campo, hojas de evaluación del estudiante, notas de evolución de cada visita y contenidos del grupo focal. Resultados: Datos recogidos indican que la intervención ayudó a los pobladores a sanar las secuelas que dejó el desastre, sin embargo, se encuentra que por falta de aceptación y compromiso de los pobladores, poco apoyo del director de la escuela y escaso esfuerzo de los líderes hubo que cancelar la ayuda. Conclusiones: El trabajo realizado por los estudiantes permitió que los pobladores pudieron volver a la rutina de su vida cotidiana, principalmente los niños que lograron reintegrarse a la escuela con mayor confianza y conociendo las medidas preventivas que proporcionaba la CONRED en caso de ocurrir un desastre.
\end{abstract}

PALABRAS CLAVE: Intervención, desastre, Psicología Comunitaria.

\section{ABSTRACT}

During 2010, the community of San Vicente Pacaya located in Escuintla State, Guatemala, was stricken by two natural disasters: the eruption of a volcano in the area called Volcán de Pacaya and the Agatha Storm. In order to ease the impact of such events, a post-disaster community intervention plan (PDCIP) was applied by a group of Psychology students in San Francisco de Sales during 2012 and 2013. This papers aims to assess the collective and individual effects of the PDCIP. Data were collected individually and in groups through diaries, student's evaluation sheets, registers of the evolution in each visit and focus group interviews. Results show the PDCIP did help inhabitants in the area overcome the psychological effects of the disasters. Unfortunately, the plan was cancelled due to lack of acceptance and commitment of some inhabitants in the area, the small effort from the community leaders and minor support on behalf of the principal of the school. In conclusion, thanks to the PDCIP, the members of the community retook their daily routines. Children went back to school, experiencing a sense of security after the natural disaster protocol training received by CONRED.

KEY WORDS: Intervention, disaster, community psychology. 
INTRODUCCIÓN

El 27 de mayo de 2010, el Volcán de Pacaya provocó la caída de arena y ceniza sobre el Lago de Amatitlán, Villa Nueva y el valle central de la ciudad de Guatemala. Un día después, entra la tormenta tropical Agatha al territorio guatemalteco, alcanzando las lluvias un volumen de 350 a $550 \mathrm{~mm}$ en 24 horas, un índice histórico en Guatemala (Segeplan, 2010), afectando, entre otros, al Municipio de San Vicente Pacaya.

Dicho municipio se encuentra situado en la parte Noreste del departamento de Escuintla, en la Región V o Región Central. Cuenta con una extensión territorial de 236 kilómetros cuadrados y se encuentra a una altura de 1,680 metros sobre el nivel del mar, por lo que generalmente su clima es Templado. Se encuentra a una distancia de 27 kilómetros de la cabecera departamental de Escuintla. La municipalidad es de cuarta categoría, el municipio cuenta con un pueblo, que es la cabecera municipal San Vicente Pacaya, cinco aldeas, seis caseríos y siete parajes. Las aldeas son: Bejucal, El Cedro, El Patrocinio, Los Chagüites y San Francisco de Sales, de estas, se decidió hacer intervención en las de San Francisco de Sales y San José Calderas. Como antecedente, luego de los desastres naturales, el departamento de Responsabilidad Social Universitaria (RSU) de la Universidad Rafael Landívar apoyó con atención psicosocial a la comunidad de El Pepinal, una de las aldeas pertenecientes a San Vicente Pacaya. Durante el mes de octubre de 2011 se intervino desde la Escuela Oficial Rural Mixta de San Antonio, El Pepinal. Se trabajó con niños, adolescentes y adultos en diferentes momentos. La conclusión a la que se llegó era que los miembros de la comunidad no sólo estaban sufriendo las consecuencias de los efectos de los desastres naturales sino también padecían desórdenes psicológicos, como ansiedad, represión de sentimientos y creencias disfuncionales. A partir de varias intervenciones se concluyó que las personas de esta comunidad y otras aledañas, como las elegidas, estaban necesitadas de intervención psicosocial.

San Francisco de Sales es una aldea que se encuentra dentro de los límites del Parque nacional del Volcán de Pacaya. La comunidad posee aproximadamente 1800 habitantes y está conformada por 258 familias que residen en la localidad. La tasa de crecimiento poblacional es menor del 2.5 $\%$. Fue establecida como aldea en 1906.

San José Calderas, según datos del último censo de octubre de 2011, está constituido por una población total de 1,042 habitantes, divididos en 285 familias, con una extensión territorial equivalente a 9 kilómetros cuadrados. Su composición étnica es en su mayoría ladinos y en un porcentaje menor indígenas. Las tasas de nacimientos son de 12 a 20 nacimientos por año y de mortalidad de 2-5 habitantes al año. La religión predominante es la católica.

Las intervenciones planteadas fueron realizadas desde el modelo de la Psicología Comunitaria. En términos generales, la práctica de la psicología social comunitaria en Latinoamérica ha estado orientada hacia la transformación social, que pretende descubrir otra perspectiva en las comunidades y en los actores sociales. Su objetivo es apuntar a que sean los mismos pobladores los que obtengan el fortalecimiento de su capacidad de decisión, el control de sus propias acciones y la responsabilidad por sus consecuencias (Parisí, y Marín, 2012; Parra, 2008). La psicología se introduce en las comunidades y redefine la salud mental de las mismas tomando en cuenta los contextos históricos particulares, que se contraponen al concepto de salud mental de la psicología individual dominante. La contraposición se da especialmente en el funcionamiento de las políticas de salud que se desarrollan desde las cúpulas de poder de los 
gobiernos y que a su vez están orientadas por lineamientos que responden a intereses económicos y que buscan preservar un orden ya establecido socialmente.

La psicología comunitaria surge con cierta timidez, como menciona Sánchez (2001), en los años setenta y lo hace con el objeto de dar atención a las condiciones de vida y las demandas emergentes de la población de los sectores más desamparados o vulnerables. En América Latina se desarrolla desde las experiencias ligadas a las realidades locales, a los fracasos cuando la comunidad no fue involucrada en los procesos y a los logros cuando se le involucra en la investigación y en la resolución de sus problemas.

modelo que más se ha difundido en la intervención psicosocial es aquel que una comunidad trabajando con un equipo de psicólogos asume el control a través de la participación y de la toma de decisiones para resolver un problema que les afecta.

Su origen está ligado al cambio de paradigma de la psicología social tradicional, Parra, (2008). Su base psicosocial, así como el uso que hace de procedimientos, técnicas, instrumentos y métodos provienen de la Psicología Social, como indica Montero, (1984). Los ejes en torno a los que trabaja son las necesidades y preocupaciones de las poblaciones, el rol profesional del psicólogo como agente de cambio social, la utilización de conceptos sociológicos y el interés por contribuir a la solución de problemáticas concretas.

Las intervenciones que se dieron inicialmente en el 2010 fueron una serie de actividades que pretendían conocer en qué condiciones estaban las comunidades luego de los desastres naturales sufridos. Con respecto a este tipo de intervenciones Pineda, y López-López, (2010) refieren que estas estrategias de atención son una forma de entender la crisis. Una de las principales metas que se establecieron desde el inicio de la intervención fue el poder propiciar el cambio en las relaciones de poder horizontales y generar el sentido de solidaridad, responsabilidad y pertenencia del individuo de la comunidad. Todo ello debe propiciar que el rol de psicólogo sea de acompañamiento, de manera que los miembros de las comunidades sean quienes tomen las decisiones que les atañen. Este modelo, como indica Ulivarri (2010), enfrenta varias premisas, una postura ética que es vital en el contacto con los miembros de la comunidad y que impone responsabilidad y compromiso. El trabajo realizado ha sido sustentado desde la convicción de que las comunidades tienen recursos y potencialidades para identificar y resolver sus propios problemas. Las competencias multiculturales que tenemos que incluir en las intervenciones tienen que estar orientadas a enfatizar la integración de los elementos de la cotidianidad comunitaria teniendo en cuenta su ideología, cultura, identidad y sus recursos tanto materiales como especialmente humanos para enfrentar una crisis.

Las crisis que se generan a partir de un desastre natural son de varias índoles. Para entender mejor qué es una crisis se puede conceptualizar como un estado temporal de trastorno y desorganización, en el que la situación desborda la capacidad de afrontamiento. Las personas se enfrentan a no poder solucionarlo u obtener resultados positivos. Las características que tiene que tener son: afectar a muchas personas a la vez, tiene un carácter repentino, un impacto poderoso, percepción de incapacidad de control, pérdida de confianza de las víctimas y persistencia de los efectos (Pineda y López-López 2010).

Las fases de la vivencia de la crisis pueden ir desde el desorden, que es el conjunto de conductas emitidas después del desastre. Luego aparece una etapa de negación, que es un intento de omisión de la realidad latente por el desastre. La intrusión es un abarrotamiento de ideas y sentimientos de dolor. La traselaboración 
significa que las personas expresan pensamientos y sentimientos que habían permanecido guardados y que ahora pueden tener un nuevo significado. La terminación es un nuevo comienzo, es la adaptación de la experiencia a la historia de vida (Pineda y López-López 2010).

Gaborit, (2006), ha propuesto un modelo explicativo de las reacciones traumáticas ante el desastre, la que incluye diferentes factores identificables que determinan la posibilidad de reacción. Este modelo apunta a que la severidad de la reacción 0 la elaboración del trauma tienen que ver con el individuo y sus respuestas. La severidad a su vez, con la que las personas reaccionan dará la pauta del tiempo y curso que durará la recuperación.

Los tres medidores sociales que propone este modelo son: El apoyo social, cuanto mayor sea la red de personas 0 instituciones que apoyen más rápido es la recuperación. Las estrategias de afrontamiento que son las acciones dirigidas a la solución que tienen un impacto emocional por el efecto del trauma ante el evento. El significado que cada individuo le da al acontecimiento (Montero, 2003).

En el caso del apoyo social, el papel de la mujer es preponderante. Las mujeres ocupan un lugar importante en la participación, ya que organizan a sus grupos familiares en función de la protección de los hijos, huir del peligro, buscar alimentos y el más importante dan soporte afectivo y apoyo. Los sectores más afectados son los niños y los ancianos, lo cuales se convierten en prioridad cuando las mujeres son encargadas en el papel de "cuidadoras" dando soporte material y psicológico. La mujer por tanto tiene una posición de liderazgo en la reconstrucción de la vida cotidiana luego de un desastre natural (Magaña y Silva-Nadales, 2010).

Tradicionalmente las intervenciones en desastres en Guatemala se han realizado a través de voluntariado de profesores y estudiantes que se ofrecen para asistir a las comunidades a dar el apoyo. La mayoría de las veces se hace por medio de alguna institución pública o las universidades del país, tanto la San Carlos de Guatemala, Universidad estatal, como las privadas (Duque, Cabrera, García y Paz, 2011). No se ha reportado la evaluación del impacto en ninguna de las intervenciones revisadas. En este contexto, el objetivo de nuestra investigación fue evaluar el efecto de la intervención realizada en San Francisco de Sales mediante diversas técnicas de recolección de información, centradas principalmente en la vivencia de los sujetos participantes.

\section{METODO}

Dentro del Pensum de la carrera de Psicología con énfasis en Clínica del departamento de Psicología de la Universidad Rafael Landivar, se decidió abrir un espacio de trabajo desde el curso de Intervención Psicológica. Este curso tiene como objetivo ser un espacio de formación continua para los estudiantes de manera que combinen actividades teóricoprácticas e inicien la aplicación de los conocimientos básicos adquiridos durante los primeros tres años de la carrera. Este curso tiene una duración de un año lectivo y está dividido en por tres módulos, primer semestre, interciclo y segundo semestre, que abarcan todo el año.

La metodología utilizada fue sistematizar la experiencia en varios instrumentos:

a) El diario de campo de cada uno de los estudiantes, el cual entregaban durante el período de clase posterior a la visita semanal. Los lineamientos para la realización del mismo era escribirlo a dos columnas, del lado izquierdo llevar un record histórico de los sucesos de la visita, tomando en cuenta desde la salida de la universidad hasta el regreso. En la 
columna derecha anotar qué les generó la experiencia de la visita. La redacción se pidió en forma de ensayo para que la elaboración de pensamientos y sentimientos fuera más efectiva.

b) Las notas de evolución grupal, que se redactaban por cada visita e intentaba recoger los siguientes datos: principales problemas encontrados en la comunidad que se tomaban como temas a tratar 0 asuntos a trabajar en las próximas visitas. Personas de contacto, ya sea líderes de la comunidad con quienes hay que entrar en contacto o que intervinieron en las actividades programadas. Problemas encontrados para el estudiante, dificultades para el acceso, flexibilidad y apertura de los miembros de la comunidad.

c) El plan sugerido de seguimiento. Este instrumento fue realizado en el 2010 por González, A. durante las visitas que se realizaron a comunidades del altiplano de Guatemala durante la tormenta Agatha.

d) Una evaluación individual, instrumento que fue elaborado por las estudiantes del curso de Intervención Psicológica sección dos, intervención al Volcán de Pacaya de 2012. El mismo instrumento fue utilizado por las estudiantes del curso 2013.

e) Realización de un grupo focal con los miembros del COCODE de la comunidad de San Francisco de Sales, una de las dos comunidades intervenidas. El mismo se llevó a cabo el día viernes en las instalaciones de la Telesecundaria. Dio inicio a las nueve horas y terminó a las once y treinta. Los datos fueron transcritos por una estudiante del grupo, utilizándose luego el método de "la mesa larga" de Krueger y Casey (2000) para organizar los datos y encontrar los enunciados de significado. Se utilizaron papelógrafos y en cada uno se puso como título los temas que surgieron de los enunciados, nos basamos en las preguntas elaboradas como guía para el grupo focal. Luego se organizó la información de los hallazgos, en una tabla se colocaron los indicadores encontrados en las trascripciones, en la segunda columna, el criterio y en la tercera, las frases encontradas para sustentar el indicador.

\section{Hallazgos}

Los problemas con los que los estudiantes se fueron encontrando a lo largo del trabajo del año fueron las mismas secuelas que dejo la erupción del volcán y las lluvias torrenciales de la tormenta Agatha. La lluvia de piedras de fuego que lanzó el volcán ocasionó un sin número de destrucción en las viviendas de la aldea. Muchos techos de lámina y varias paredes de concreto fueron demolidas por la cantidad de piedras de todo tamaño que lanzó el volcán. En San Francisco de Sales hubo varias viviendas destruidas, una construcción afectada fue el salón comunal, que dejó a la comunidad sin un espacio físico en donde reunirse. Está pérdida también imposibilitó a los estudiantes contar con un lugar de buen tamaño para realizar los talleres y conferencias, principalmente a padres de familia.

Otro problema que azota a todo el territorio guatemalteco es la delincuencia, el robo de los cables de electricidad por personas ajenas a la comunidad, según los pobladores, ha dejado a la escuela sin ese servicio, lo que no permitió el uso de tecnología para las intervenciones. 
La idiosincrasia de los pobladores de las comunidades, su nivel de instrucción académica, las cualidades propias dan a la actividad de la aldea, como a sus pobladores un ritmo propio que desde una perspectiva nuestra, ajena a esa realidad, es incomprensible. El tiempo parece tener otra connotación, muy diferente a la nuestra. Se aprendió a planificar las actividades, acordándolas con sus miembros y a pesar de ello, tenían sus prioridades y no asistían a las actividades programadas. Por ello, en varias oportunidades se tuvo que suspender lo que se había preparado, ya sea porque no había asistencia, la escuela estaba cerrada por razones, a nuestro juicio injustificadas, pero con sentido para ellos. Las personas que buscaban atención individual tenían otras cosas que hacer dejando de lado el compromiso adquirido con el estudiante y simplemente no se presentan a su cita. Las razones eran múltiples, con sentido para los pobladores, aunque sin sentido para nosotros, que asistíamos puntualmente todas las semanas, los estudiantes, los profesionales encargados del programa de RSU y la docente.

El aporte de los estudiantes también tuvo sus contratiempos, tales como falta de compromiso, olvido de materiales, poco apoyo a la coordinación fueron algunas de las razones por las que el trabajo no se concretó como se esperaba. Los estudiantes son jóvenes que están en formación y que en su inmadurez, en ocasiones se les dificulta vincularse con su rol profesional.

Se llevó a cabo la elaboración de un grupo focal con la finalidad de conocer la opinión que tenían las personas de la comunidad con respecto a la intervención que estaban realizando los estudiantes de Psicología Clínica. En base a la transcripción que se obtuvo a partir del grupo focal se encontraron trece indicadores, los cuales se describen a continuación detalladamente.
Secuelas del desastre: la erupción volcánica del año 2010 dejó secuelas de diversa índole a las personas de la comunidad. Entre ellas está el estrés posttraumático debido al temor constante de que una situación de este tipo se vuelva a presentar en cualquier momento. También se presentaron casos de niños que se orinaban en sus camas por las noches después del desastre ocurrido. "Primero porque a raíz de la erupción los niños quedaron traumados, en mi opinión." Algunas personas presentaron temor de volver a la comunidad y prefirieron ir a vivir a otros lugares, la infraestructura se vio afectada significativamente a lo largo de toda la comunidad. Hubo daño a cultivos y en general la economía de los habitantes se vio seriamente afectada.

Acompañamiento: En base a las problemáticas de distinta índole que se viven en la comunidad. Los estudiantes de Psicología, en el presente año, dieron seguimiento al plan de intervención que se comenzó el año pasado. Con este seguimiento, las personas de la comunidad se sentían apoyadas y agradecidas con los estudiantes, ya que ellos consideran que el trabajo realizado fue de gran ayuda. "Y otro es de que les gusta ayudarnos, les gusta darnos su apoyo para todos los niños y tanto a los niños como a las personas grandes verdad, ustedes siempre están ahí para apoyarnos en cualquier cosa que necesitemos." Apoyando a superar la situación vivida después de la erupción y la tormenta. Este acompañamiento fue uno de los principales logros durante esta intervención. Con el tiempo, las personas lograron tomar de nuevo el curso de sus vidas, los niños ya no tenían tanto temor de volver a la escuela, en general las personas se sienten más preparadas en el momento de volver a enfrentar un desastre similar.

Por otro lado, en base al trabajo que los estudiantes realizaron durante este año, algunas personas y líderes de la comunidad tenían el deseo de que los estudiantes de 
Psicología Clínica siguieran dando continuidad a esta intervención, principalmente porque algunos de ellos lograron sentir la suficiente confianza en ellos como para comunicarles alguna situación para la cual necesitaran de su ayuda.

Actitud de la comunidad ante los estudiantes: varias personas expresaron sus muestras de agradecimiento por el trabajo que se elaboró en la comunidad ya que aseguran sentirse mejor consigo mismos e incluso felices. Por otro lado, expresaron sus sentimientos de pesar ante diversas situaciones que se presentaron durante el transcurso de la intervención, como lo fue la falta de interés de algunos miembros de la comunidad en cuanto al trabajo que los estudiantes realizaban, principalmente dentro de la escuela. Estas situaciones contribuyeron a que las autoridades de la Universidad tomarán la decisión de realizar el cierre del proceso de intervención.

Algunos miembros del COCODE, padres de familia y maestros consideraban que la labor de los estudiantes en la comunidad fue benéfica y lamentaban el hecho de que en ocasiones esta labor se veía interrumpida por factores como falta de compromiso de algunos miembros. En varias ocasiones los estudiantes encontraban la escuela cerrada habiendo llegado preparados con materiales para la realización de talleres 0 para las intervenciones individuales. También fue evidente que algunas personas no tenían realmente clara cuál era la labor de los estudiantes de Psicología Clínica en la comunidad y por tal razón preferían no involucrarse en las actividades. En ocasiones se debía a que las personas mostraban pena o vergüenza de hablar de su vida personal con personas ajenas a su comunidad.

Algunos miembros del COCODE consideran tener responsabilidad en la falta de compromiso de algunos miembros de la comunidad porque ellos, siendo miembros líderes y destacados de la comunidad, no se encargaron de informar y motivar a las personas. . Consideran que si ellos hubiesen apoyado más en este proceso, es probable que las personas de la comunidad se hubieran incorporado más en las intervenciones que los estudiantes estaban realizando dentro de la comunidad.

La postura de otras personas de la comunidad, según los indicadores encontrados, fue que pensaban que los estudiantes realmente no tenían nada que hacer o que no hacían nada en sus casas, que nada más iban a "molestar" o a recabar datos y que por tal razón únicamente iban a la comunidad a sentarse y a pasar el tiempo. "Ustedes son un grupo de huevonas que no tienen oficio qué hacer en sus casa y por eso vienen a chingar a la comunidad".

Disposición de la comunidad a recibir ayuda: Los estudiantes de Psicología Clínica se encontraron con diversos inconvenientes dentro de la escuela entre los que se pueden mencionar: tener prioridad a otras actividades como encuentros deportivos, a los estudiantes, en más de alguna ocasión, no se les informó que la escuela se encontraría cerrada, el director y algunos maestros no asistían a sus labores en la escuela. Por otro lado, algunos niños no asistían a la escuela y se ausentaban con frecuencia en su mayor parte por razones que se desconocieron. "Lo negativo es el director, el no da la entrada, incluso el Presidente del COCODE se ha alegado con los maestros."

Las madres de familia estaban más involucradas y comprometidas en cuanto a las actividades que los alumnos realizaban dentro de la escuela. Resulta evidente que la participación de los padres de familia no fue tan activa como la de las madres, esto en gran medida se debe a que ellos tienen un horario de trabajo con el cual deben 
cumplir para poder ganar el sustento de sus familias, algunos trabajan en el campo y otros tienen empleos fuera de la comunidad. Elaboración de talleres: para el día de las madres, los estudiantes de Psicología Clínica llevaron a cabo la realización de un taller dedicado a las madres de la comunidad. Dicho taller fue de su agrado ya que dieron gracias a los estudiantes por dedicarles un espacio y tiempo en el que ellas se olvidaran de sus cotidianidades y así pudieran convivir, expresarse y reflexionar con otras madres. "Si porque incluso hubieron mamas que me dijeron que habian estado bien felices tal vez porque ellas no habian tenido actividades". Además hicieron énfasis que anteriormente la escuela realizaba actividades de este tipo pero hace ya algunos años no se realizaba ninguna actividad de este tipo, porque generaba disputas.

Los talleres resultaron de gran beneficio para los alumnos de la escuela de la comunidad ya que la temática de estos se enfocaban en las necesidades que los maestros indicaron al inicio del año. Los temas fueron: respeto, autoestima, fortalezas y debilidades, sexualidad, entre otros. Se evidenciaron cambios positivos en su comportamiento, más motivación para ir a estudiar, tener más conocimiento $y$ extender su interés a temas de diversa índole, aplicar lo que ellos aprendieron en los talleres a su vida diaria, etc. El hecho de que los talleres se impartieran de una manera que fuera dinámica y que fomentara la interacción y apoyo entre sus participantes hacía que estos fueran más llamativos y efectivos para los alumnos que participaban.

Ayudar a manejar el miedo: con las intervenciones realizadas por el grupo de estudiantes del año pasado se logró trabajar principalmente en el estrés post-traumático que había generado la erupción del Volcán de Pacaya y la tormenta tropical Agatha en los habitantes de la comunidad. Se impartieron talleres relacionados a los desastres naturales y atención de casos clínicos que ameritaban atención personalizada. Los estudiantes del presente año dieron seguimiento a este trabajo aunque y ampliaron la intervención, se tomaron en cuenta otros temas que también demandaban atención dentro de la comunidad. Fue evidente que tales intervenciones tuvieron aspectos positivos en los habitantes de la comunidad principalmente en los niños de la escuela.

Posterior al desastre los alumnos no mostraban deseos de asistir a la escuela por el miedo a que una situación de este tipo se volviera a repetir, no obstante con el pasar del tiempo, fueron recuperando la confianza y la tranquilidad y a la vez aprendieron formas de cómo actuar previo, durante y después de un desastres natural. "Los niños no querían venir porque decían que dé a rato a rato podía hacer otra erupción, como cuando ustedes comenzaron a venir". Esto se podría considerar como uno de los aportes más significativos que los estudiantes de Psicología Clínica llevaron a cabo durante estos dos años de intervención.

Ayudar a socializar: previo a la intervención de los estudiantes los miembros de la comunidad presentaban falta de confianza ante personas ajenas a su comunidad y por lo tanto no mostraban interés en involucrarse en tareas que delegaban miembros de organizaciones que provenían de otros lugares. "Aquí hay muchas personas que no les gusta participar en actividades, son bien tímidos, por eso hablan eso ellos verdad". Su pensamiento era que estas personas podían llegar con malas intenciones o que podían difundir la información la a otras personas y, por lo tanto, no veían algún beneficio en las actividades preferían no involucrarse en las mismas. Poco a poco este comportamiento fue cambiando, ya que los estudiantes de Psicología Clínica se dieron cuenta que hubo manifestaciones de más apertura por parte de los miembros de la comunidad 
involucrándose a las actividades que se realizaron, lo cual se considera es otro de los aportes más positivos que se logró en esta intervención.

Ayudar a sanar enfermedad: después de la erupción del volcán los niños de la comunidad manifestaron un desajuste emocional, algunos presentaban miedo, se asustaban con facilidad, enuresis (orinaban la cama); entre otros. Por lo que, la intervención del grupo que trabajó en el 2012, logró ayudar emocionalmente a niños y niñas para lograr tener un mayor control sobre ellos mismos a través de técnicas psicológicas que aportaron la ayuda necesaria de forma individual, por medio de la psicoterapia y a través de los talleres realizados dentro de cada clase.

"Y otro también que les han ayudado en que los niños también que muchos quedaron con un defecto mal verdad con 10 del volcán este quedaron como enfermos, ellos se orinaban en la cama entonces eso tuvimos nosotros con el grupo del año de la vez pasada tuvimos eso de estarlo hablando verdad porque las mamas nos contaban $y$ ellos fueron los que también les ayudaron y todo eso les fue ayudando como a que sacaran ese miedo verdad y ellos también tal vez por miedo ellos se orinaban en la cama por no salir verdad y en todo eso les han ayudado bastante."

Acciones para realizar en caso de un desastre: el desastre natural de la erupción volcánica provocó en las personas de la comunidad el no saber actuar en casos de emergencia porque fueron invadidos por el miedo y susto de lo que podía pasar. A consecuencia de lo mismo, los estudiantes observaron la necesidad de realizar talleres preventivos que brindarán los conocimientos necesarios para que las personas pudieran actuar en casos de emergencia ante una erupción volcánica y/o cualquier desastre natural. "Como actuar en casos de emergencia, tener cosas preparadas que antes no sabíamos, ahora ya sabemos que tenemos que tener."

Necesidad de ayuda psicológica: Durante el año 2012 se llegó a la comunidad con el fin de brindar intervención pos desastre a consecuencia de la erupción del volcán. Se inició el trabajo psicológico desde el año pasado por medio de planificaciones de talleres; de tal manera que se brindarán las herramientas necesarias a las personas, niños y niñas de la comunidad. Posteriormente, se trató de abarcar a través de pruebas proyectivas aplicadas directamente a los alumnos de la escuela y dependiendo de los resultados que las mismas arrojaran se iniciaba un proceso terapéutico según las necesidades de cada persona.

Durante el año 2013 la posibilidad de ayuda psicológica estaba abierta para todas aquellas personas que se acercarán a buscarla para equilibrar sus emociones, sentimientos y pensamientos. Sin embargo, contextualizar la aceptación de un nuevo grupo de estudiantes no era algo sencillo de asimilar por parte de la comunidad; así como el entendimiento del verdadero significado de la palabra psicología, por lo que la estrategia de ayuda psicológica fue rechazada por una buena parte de la comunidad San Francisco de Sales, lo cual ocasionó la finalización de la intervención terapéutica.

Labor de los estudiantes en la comunidad: El objetivo esencial de la intervención comunitaria fue brindar, a través de los estudiantes de la carrera de psicología clínica, un bienestar emocional y cognitivo a las personas de la comunidad San Francisco de Sales.

La labor esencial de los estudiantes hacia la comunidad es brindar el servicio de ayuda psicológica; de tal manera, que todas aquellas personas de la comunidad que están atravesando algún problema personal, emocional, laboral o de cualquier índole y 
sienta la necesidad de acercarse puede hacerlo con libertad y sin ser coaccionado. "entonces ya con la ayuda de ustedes pues ya los niños pues agarraron más, ¿cómo le dijera yo? agarraron más experiencia de venir a la escuela y todo porque habian niños de que no querían venir por el miedo siempre al Volcán de Pacaya."

Seguimiento del programa: Se inició el trabajo comunitario en el año 2012 donde un grupo de estudiantes comenzó a trabajar distintas temáticas relacionadas a la intervención pos desastre de la comunidad. Dicho grupo culturalmente fue aceptado; sin embargo, la continuidad del programa para el año 2013 era darle el seguimiento necesario del trabajo comunitario que había sido iniciado por los estudiantes del año 2012, pero esto no fue posible debido a que las personas de la comunidad desconocían la labor de los psicólogos en entrenamiento. Ese proceso de intercambio de estudiantes entre un año y el otro no fue aceptado por la comunidad; por lo que no facilitó el seguimiento del programa tanto con la escuela para intervención de los alumnos, personeros de la comunidad y el mismo comité.

Timidez de los miembros de la comunidad: Muchas de las personas de la comunidad, se consideran personas introvertidas, tímidas que los lleva a ser desconfiados en un punto crítico, como para no expresarse por miedo al que dirán los demás. "Si venía un niño desconocido entonces ahi humilladitos y todo verdad, no platicaban no se hacian amigos entre ellos verdad entonces todo eso si les ha ayudado bastante". El que estas personas manejen su narrativa con estudiantes en entrenamiento es considerado como algo vergonzoso, debido a que no es aceptable culturalmente que las personas de la comunidad hablen de su vida personal, de sus problemas y emociones con alguien que para ellos es una persona ajena a la comunidad. Dentro del contexto y culturalmente no es aceptado. "Falta de confianza, en las personas de afuera podría ser."

\section{Discusión}

La Psicología en Guatemala, es una ciencia bastante joven ya que es hasta en los últimos años que ésta ha ido cobrando fuerza poco a poco y las personas en general le van dando más valor y empiezan a tener más conocimiento sobre ella. Mientras tanto, el tema de la Psicología dentro de las comunidades es algo relativamente nuevo que también poco a poco se está abriendo camino en Guatemala (Duque, Cabrera, García y Paz, 2011).

Se han llevado a cabo diversos intentos con tal de dirigir un nuevo enfoque de lo que es la Psicología dentro de las comunidades. Los profesionales en el campo de la salud mental han descubierto que tanto actuales como anteriores enfoques que se han desarrollado dentro del campo de la Psicología han demostrado ser eficaces en determinadas poblaciones, pueden no serlo para otras (Montero, 2003). Esto es debido a que muchas teorías plantean hipótesis relacionadas a aspectos íntimos y cotidianos de la vida de cada persona dentro de un contexto biopsicosocial y se han encontrado que estos enfoques que ellos han desarrollado en base a los individuos de determinadas poblaciones pueden no resultar útiles para individuos que viven en contextos totalmente distintos.

Por razones como éstas es que ha surgido la necesidad de abrir nuevos campos de investigación para comprender cómo el contexto puede variar de un lugar a otro y para saber esto incluso no es necesario salir de las fronteras de un país, sino que incluso en un mismo país pueden existir gran diversidad de contextos. Otra razón importante, aplicable en Guatemala y casi el resto de países del mundo es la desigual distribución de poder (Montero, 2003). Es evidente que en nuestro país se 
ha centrado en ciertos contextos sociales mientras que otros contextos son regidos por el poder que estos mismos ejercen. Esto los lleva a no tener acceso a bienes sociales que les garantice una mejor calidad de vida y se les limite el acceso a ciertos servicios como la vivienda, el transporte, salud, educación, etc. Esta es la característica que con más frecuencia se ha repetido en nuestro país a lo largo de los años trayendo consigo más efectos dramáticos que positivos.

Sin duda alguna, tal desigualdad ocasiona desajustes en las personas que lo viven, por tal razón es que ha surgido la necesidad dentro del campo de la psicología comunitaria de comenzar a realizar transformaciones, tanto en los grupos como en las comunidades que los necesitan para hacer un cambio positivo en sus vidas (Pineda, y López-López, 2010). Un claro ejemplo de los esfuerzos que se han empezado a llevar a cabo es lo que se presenta en este documento, ya que luego de las secuelas tanto de la erupción del Volcán de Pacaya como las lluvias torrenciales ocasionadas por la tormenta Agatha, que dejaron graves secuelas en diversos territorios alrededor del país, comenzó la iniciativa de la Universidad Rafael Landívar de llevar a cabo una intervención en los sectores que habían sido olvidados por organizaciones del estado (Universidad Rafael Landivar, 2010).Uno de estos lugares fue la Comunidad de San Francisco de Sales ubicada en el Municipio de San Vicente Pacaya.

La principal prioridad en el primer momento de iniciar la intervención fue aliviar las secuelas emocionales que presentaban las personas de la comunidad a raíz de estos dos desastres; es decir, volver a tomar el control de sus vidas (Pineda, y López-López, 2010). En el año 2012 empezó tal intervención por parte de los estudiantes de Psicología Clínica de la Universidad Rafael Landivar, la tarea de estos estudiantes era llevar a cabo acciones que orientaran a estas personas sobre cómo lidiar con las secuelas que tales desastres habían dejado en sus vidas. Por otro lado, el enfoque también se orientaba sobre cómo actuar si se volviese a presentar una situación de este tipo para estar mejor preparados.

Al parecer, se lograron bastantes cambios positivos durante este período de intervención en el año 2012, no obstante este trabajo no era suficiente pues en la comunidad también se presentaban otro tipo de problemáticas que también ameritaban atención. Por tal razón, en el año 2013 se tomó la decisión de dar seguimiento al trabajo que los estudiantes del año 2012 habían realizado dentro de la comunidad y ampliar la intervención.

Los estudiantes tenían un reto muy grande, ya que en primer lugar debían ganarse la aceptación y confianza de las personas de la comunidad. El hecho de que el año anterior, las personas hubiesen estado con otro grupo de estudiantes hacía un poco más complicada esta tarea, pues debían conocer a estos nuevos estudiantes para poder confiar en ellos.

A lo largo de su intervención se fueron dando cuenta de que en ocasiones dentro de las comunidades se cuenta con códigos y reglas que rigen su forma de relacionarse entre sí y con miembros que provienen de otros lugares. Descubrieron que no resulta tan sencillo el hecho de ser aceptados de un día para otro por los miembros de una comunidad ya que ésta resulta ser una tarea que lleva tiempo y constancia.

Durante la realización del grupo focal se encontró que algunas de las personas de la comunidad mostraban cierta reserva con los estudiantes, esto por varias razones. En primer lugar hacían referencia que para ellos es difícil el hecho de compartir su vida con personas ajenas a su contexto ya que su principal temor era que lo difundido a los estudiantes luego se supiera por toda la 
comunidad y se convirtiera en una especie de rumor o "chisme". Además fue evidente que algunos miembros no daban valor 0 importancia al trabajo que los estudiantes desempeñaban dentro de la comunidad, en parte era por falta de compromiso y por otro lado, por la falta de información sobre el trabajo y el enfoque que tenían los estudiantes para trabajar dentro de la comunidad. Para ellos simplemente resultaba difícil el hecho de compartir su vida o trabajar al lado de personas que no pertenecen a su contexto y por tal razón prefieren no hacerlo.

Este tema está más arraigado a la idiosincrasia de los pobladores, ya que son es costumbre entre ellos de querer enterarse de todo lo que pasa y si tienen alguna información sobre un miembro de la comunidad esparcirla, lo que es conocido en Guatemala como "chisme". Por tal razón, no confían ni en sus vecinos y comunitarios, mucho menos en personas de fuera. "Si venía un niño desconocido entonces ahí humilladitos y todo verdad, no platicaban no se hacian amigos entre ellos verdad entonces todo eso si les ha ayudado bastante".

En cuanto a temas relacionados a sexualidad se encontró dificultad de apertura por parte de los miembros de la comunidad. Es un tema del que para ellos aun resulta difícil hablar y más aun con personas ajenas a la comunidad. Es evidente que hay una alta incidencia de embarazos en adolescentes y paternidad a temprana edad. Otro aspecto relevante es que hombres como mujeres a lo largo de su vida pueden tener más de alguna pareja y concebir hijos. Muchas de las parejas no llegan a contraer matrimonio.

Se presenta el tema de incesto dentro de la comunidad, un tema que está inmerso en el silencio. Sin duda alguna, lo que en otros contextos sociales podría parecer como algo totalmente inaceptable, para muchos miembros de la comunidad puede ser visto como algo dentro de la norma. Por lo tanto, forma parte de sus costumbres y no necesariamente está fuera de lugar.

Es a través de situaciones como estas en donde se percibe reserva de estos miembros ante temas que ellos aún no se encuentran preparados para tratar. Esto hace ver a los Psicólogos Clínicos que el trabajo en una comunidad, es un trabajo complejo en el que se necesita conocer el contexto, historia, cultura, creencias, economía, etc. para poder llevar a cabo una intervención que se ajuste a las necesidades que la comunidad presente en el momento que la misma solicita. El trabajo debe llevarse a cabo bajo su propio ritmo ya que la sugerencia de temas puede tener resultados indeseados 0 inesperados por parte de los miembros.

Los líderes dentro de una comunidad cumplen con una función muy importante la cual es sintonizar las relaciones dentro de los miembros así como llevar a cabo la organización de eventos importantes, reuniones, comisiones, entre otras. El funcionamiento de una comunidad no podría ser el mismo si no tuviera el apoyo de estos líderes. El líder se encarga de velar por los intereses, encontrar solución a los problemas, plantear propuestas para el mejoramiento de las condiciones de vida de la comunidad además de tener contacto con organizaciones externas que apoyen al funcionamiento de la misma.

Los líderes cumplieron una función muy importante para los psicólogos en entrenamiento que se adentraron en el trabajo con una comunidad. Ayudaron a establecer las necesidades que la misma presentaba y proporcionaron la conexión de los estudiantes con los demás miembros de la comunidad, en pocas palabras fueron quienes establecieron la relación de confianza entre ambas partes. Sí este encuentro no se hubiera dado, hubiera sido prácticamente imposible entrar a la comunidad para trabajar con las personas. 
Los líderes de la comunidad de San Francisco de Sales mostraron interés y compromiso en cuanto al trabajo que los estudiantes desempeñaron dentro de su comunidad. Eran conscientes del beneficio que tanto ellos como los estudiantes obtenían a partir de la elaboración de talleres y atención de casos clínicos. Consideran que hubo aportes significativos por parte de los estudiantes que dieron lugar a ciertos cambios dentro de su comunidad, entre estos se encuentran el superar las secuelas y trauma que dejó tras de sí la erupción volcánica junto con la tormenta tropical. Por otro lado, el beneficio que se obtuvo a través de los talleres ya que los alumnos de la escuela perdieron el temor de volver a su centro de estudios, además de aprender cómo actuar ante una situación de riesgo: antes, durante y después de la misma.

Por otro lado, le dieron valor al seguimiento del trabajo que se inició el año pasado ya que veían la constancia y compromiso de la Universidad, lo cual los hacía sentir más seguros. Tanto líderes como algunos maestros eran conscientes de que el trabajo de los estudiantes en ocasiones se vio interrumpido por acontecimientos que estaban fuera de sus manos, como el hecho de que la escuela, estuviese cerrada cuando ellos asistían los días viernes. La falta de compromiso del director, falta de apertura por algunos miembros y también falta de dirección por parte de los mismos líderes, fueron algunos de los problemas que se dieron.

Tanto maestros, líderes, padres de familia, como otros miembros de la comunidad lamentaban el hecho de que las autoridades de la Universidad Rafael Landívar se vieran en la necesidad de llevar a cabo un cierre de la intervención en dicha comunidad debido a las razones que anteriormente se acaban de describir. No obstante, se sabe que hubo aportes significativos dentro de la comunidad en el período de tiempo que se trabajó dentro de ella y también está el hecho de que los estudiantes lograron ampliar su perspectiva en cuanto a las implicaciones que tiene el trabajo de la Psicología dentro del ámbito comunitario.

\section{CONCLUSIONES}

Los hallazgos de este trabajo muestran un espectro más amplio de la intervención comunitaria a través de talleres, la aplicación de pruebas proyectivas e inició de terapias individuales que se llevó a cabo en la Comunidad San Francisco de Sales ubicada en el municipio de San Vicente Pacaya.

Las intervenciones que se dieron durante el 2012 y 2013 ayudaron a la comunidad a superar las secuelas de la erupción volcánica y la tormenta Agatha, permitiendo que la comunidad volviera a su ritmo normal de vida, especialmente que los niños volvieran con tranquilidad a la escuela.

Entre las situaciones negativas que justificaron el cierre de la intervención en junio del 2013 en la comunidad fueron: la falta de aceptación y compromiso por parte de las personas de la comunidad, falta de interés por parte del director de la escuela y falta de apoyo por parte de los líderes del COCODE.

El reto que la psicología tiene es el de capacitarse y entrenarse académicamente para abrir campos de intervención en las comunidades del país y aportar, desde su idiosincrasia los cambios necesarios para que vivan con mayor seguridad en caso de desastres.

El trabajo realizado por los estudiantes de ambos grupos, 2012 y 2013 fue realizado con mucho compromiso y profesionalismo. El carácter humanístico que le impregnaron dio los resultados esperados. Los pobladores pudieron volver a la rutina de su vida cotidiana, principalmente los niños que lograron reintegrarse a la escuela con 
mayor confianza y conociendo las medidas preventivas que proporcionaba la CONRED en caso de ocurrir un desastre.

\section{REFERENCIAS}

Asencio, D., Arriola, M., Ballesteros, A., Del Pino, P., Maldonado, J., Méndez, D., Montenegro, E., Pedroso, M., Rayo, A., Rivas, A., Solis, M. (2012). Informe final: San Francisco de Sales. Documento inédito. Guatemala.

Duque,V., Cabrera, García y Paz, (2011). Capítulo 10. Entre realidad y utopía: Psicología social comunitaria en Guatemala. En Montero, M \& SerranoGarcía (comps.) Historias de la psicología comunitaria en América Latina. Participación y transformación, (págs. 237-260). Buenos Aires, Argentina, Paidós.

Gaborit, M. (2006). Desastres y trauma psicológico. Pensamiento Psicológico, 2, 15-39.

Magaña, I. y Silva-Nadales, S. (2010). Catástrofe, subjetividad femenina y reconstrucción: aportes y desafíos desde un enfoque de género para la intervención psicosocial en comunidades afectadas por el terremoto. Terapia Psicológica, 28,2, 169-177.

Montero, M. (2003). Teoría y práctica de la psicología comunitaria. La tensión entre comunidad y sociedad. Paidós, Argentina.

Montero, M. (1984). La psicología comunitaria, principios y fundamentos teóricos. Revista Latinoamericana de Psicología, 16, 3, 387400.

Parisí, E. y Marín L. (2012). Psicología social comunitaria en Latinoamérica y la situación en Argentina. Revista Diálogos, 3, 1, 727.

Parra, M. (2008). La psicología comunitaria en América Latina. Poiesis, Revista electrónica de psicología social, 15. Disponible http://www.funlam.edu.co/revistas/index.ph p/poiesis/article/view/287/276

Pineda y López-López, (2010). Atención Psicológica Postdesastres: Más que un "Guarde la calma". Una Revisión de los Modelos de las Estrategias de Intervención. Terapia Psicológica, 28, 2, 155-160.

Sánchez, E. (2001). La psicología social comunitaria: repensando la disciplina desde la comunidad. Revista de Psicología, X, 002, 127-141.

Segeplan y Conred, (2010). Evaluación de daños y pérdidas sectoriales y estimación de necesidades ocasionados por el paso de la Tormenta Tropical Agatha y la erupción del Volcán de Pacaya. Resumen preliminar. Gobierno de Guatemala, Guatemala. Recuperado el 19 de mayo 2013 www.segeplan.gob.gt/.../resumen_ejecutiv o_evaluacion_Pacaya_Agatha

Universidad Rafael Landivar, (2010). Política y manual de incorporación de la RSU, Facultad de Humanidades. Universidad Rafael Landivar, Guatemala. 